the $\mathrm{f} 2$ group of RNA phages, for example, has only three genes, and $\varphi$ X 174 or fd single stranded DNA phages code for about eight genes. These phages must therefore rely almost entirely on functions of their host bacterium. Phage T7, however, which has a duplex DNA genome consisting of nineteen genes, itself codes for several of the activities needed for its infectious cycle, but is not as complex as the larger phages.

This phage has already produced surprises. Gene 1 controls the expression of most of the phage genes; mutants which lack the gene 1 protein product can only make the three earliest T7 proteins. The obvious explanation seemed to be that this gene specifies a phage $\sigma$ factor (as do comparable genes in the T-even phages) which directs the host cell RNA polymerase to transcribe the late genes of the phage. But T7 differs from the other phages because gene 1 proved to code for a new RNA polymerase, with a specificity for the $\mathrm{T} 7$ genes.

Other genes are concerned with specifying the enzymes required to replicate the phage, such as a DNA replicase and a polynucleotide ligase. Genes 3 and 6 are concerned with the degradation of host DNA which provides the nucleotides needed for the synthesis of new phages. The protein specified by gene 3 is an endonuclease which seems to attack both single stranded and double stranded DNA, and Center and Richardson report some of its properties in two articles in the Journal of Biological Chemistry (245, 6285, 6292; 1971).

The enzyme shows a strong preference for single stranded DNA, which it degrades about 150 times faster than duplex DNA. Its attack on duplex DNA generates single strand breaks, and it is not yet clear just how these are converted into the double strand breaks which are needed to split the DNA. And in vitro, at any rate, the enzyme is as active with T7 DNA as template as it is with that from the host Escherichia coli. How is the behaviour of the enzyme in degrading only T7 DNA in vivo to be reconciled with this apparent lack of discrimination? One possibility is that the product of gene 6 , which also seems to be involved in degradation of host DNA, may confer some specificity on the gene 3 endonuclease. Another is that the degradation may take place so soon and so rapidly after the entry of the phage into the bacterium that only host DNA is available for attack at that time; this implies that the endonuclease must be inactivated in some way before replication of T7 DNA generates more substrates for it. At any rate, the gene 3 product has now been unambiguously identified, even if the way it works in vivo is not quite clear, and another gene can be ticked off on the list of those whose products are known.

\title{
The Helper and the Helped
}

SoRTING out the relationships and interdependence of animal leukaemia and sarcoma viruses is, these days, a chief preoccupation of tumour virologists; for the genome of one RNA cancer virus can be enclosed in the envelope of, and assume, the host range of a second virus. Moreover, many tumour viruses are defective; they have become dependent on the help of a second virus in order to transform and replicate in some hosts. In next Wednesday's Nature New Biology, Eckner and Steeves describe just such a situation.

In 1956, Friend isolated from Swiss mice a virus preparation which, when inoculated into mice, either induces an erythroblastic leukaemia, associated with a gross enlargement of the spleen, or a lymphatic leukaemia or lymphoma associated with tumours of the thymus, the outcome of a particular inoculation depending on the strain of mice inoculated. It has since become clear that the Friend isolates contain two viruses, one of which induces lymphomas and the other induces spleen tumours or foci and erythroblastic leukaemia. But what is the relationship between these two viruses; is one a helper for the replication of the other?

Stocks of the virus which induces spleen foci always contain the virus which induces lymphomas but the converse is not the case. Further, Eckner and Steeves found that the induction of spleen tumours in some strains of mice seems to depend on the multiple infection of the spleen cells by two or more virus particles. These two facts suggest, of course, that the virus which causes spleen foci requires the help of the lymphoma virus, for that would explain both the presence of lymphoma virus in stocks of the spleen focus inducing virus and the observation that the induction of foci depends on multiple infection. But it might be argued that multiple infection somehow impairs the immune system and as a result spleen foci are able to develop. To rule out this possibility, Eckner and Steeves treated mice with cortisol to impair their immune system and found that the induction of foci remained dependent on multiple infection. When, however, stocks of the focus inducing virus were diluted into stocks of the lymphatic leukaemia virus, the induction of foci seemed to be dependent on single infections. In this situation there is clearly a great excess of helper virus; cells are always infected by it so that the induction of foci seems to depend on a single infection by the focus forming particle.

\section{Linkage of Retinal to Opsin}

RHoDopsin, a visual pigment found in vertebrate retinal rods, transduces light into a neural message. It is a chromoprotein and consists of 11-cis retinal (retinene aldehyde) and an unspecified protein, called opsin. The two are said to be linked by a Schiff base between phosphatidyl ethanolamine (PE) and retinal, which moves after exposure to light to the $\varepsilon$-amino-group of a lysine residue in the protein. Anderson, Hoffman and Hall question this assumption in next Wednesday's Nature New Biology. Is the N-retinylidene-phosphatidyl-ethanolamine (N-RPE) which has been extracted from rod outer segments, and which is dark adapted and then not exposed to light, associated with rhodopsin or not? N-retinyl-phosphatidyl-ethanolamine (N-RH $\left.{ }_{2} \mathrm{PE}\right)$ has also been extracted from rods after acidification of rhodopsin and the sodium borohydrate fixation of retinal to its original site by a secondary linkage. Anderson et al. stress that the extracts used contain much phospholipid and retinal not bound to opsin, and they suggest that the isolation of N-RPE may be derived instead from a source other than the retinal-opsin link.

To test if this is so, they measured the turnover of the rhodopsin in the outer segment disks which form part of the rods. They injected frogs with labelled
${ }^{3} \mathrm{H}$-retinol and then measured the radioactivity three days later from suspensions of their rod outer segments, subdivided into three groups, A, B and C. A was acidified, treated with sodium borohydrate, and was then subdivided into aqueous and lyophilized subgroups. Lipid was extracted from the aqueous subgroup by treatment with a $2: 1$ chloroform-methanol mixture: the separated protein contained 73 per cent of the total radioactivity. The lyophilized subgroup, which had been extracted similarly but without protein partitioning, retained 82 per cent of the activity in the protein. It was found that all the radioactive part which had been removed from the rhodopsin was associated with free retinol, and that no tritium exchange had occurred between it and opsin.

Unlike groups $\mathbf{A}$ and $\mathbf{B}$, group $\mathbf{C}$ contained rod outer segments not originally purified on an 'Agarose' column. $C$ was subdivided, one half being treated in the same way as $A$, the other like B; less than 40 per cent of the total radioactivity was associated with the protein and an average of 30 per cent was recovered with $\mathrm{N}-\mathrm{RH}_{2} \mathrm{PE}$. Anderson et al. thus confirm the presence of $\mathrm{N}$ RPE in cetyltrimethylammonium bromide solutions of rod segments, but demonstrate that this lipid forms no part of the rhodopsin chromophore. 